\title{
Multi-Objective Design Exploration and Its Application to Regional-Jet Wing Design
}

\author{
By Shigeru OBAYAShI ${ }^{1)}$ Shinkyu JeOng, ${ }^{1)}$ Kazuhisa ChIBA ${ }^{2)}$ and Hiroyuki MorinO ${ }^{3)}$ \\ ${ }^{1)}$ Institute of Fluid Science, Tohoku University, Sendai, Japan \\ 2) Japan Aerospace Exploration Agency, Chofu, Japan \\ ${ }^{3)}$ Mitsubishi Heavy Industries, Ltd., Nagoya, Japan
}

(Received August 5th, 2005)

\begin{abstract}
A new approach, Multi-Objective Design Exploration (MODE), is presented to address multidisciplinary design optimization (MDO) problems using computational fluid dynamics-computational structural dynamics (CFD-CSD) coupling. MODE reveals the structure of the design space from the trade-off information and visualizes it as a panorama for Decision Maker. The present form of MODE consists of the Kriging Model, adaptive range multi objective genetic algorithms, analysis of variance and a self-organizing map. The main emphasis of this approach is visual data mining. An MDO system using high-fidelity simulation codes, a Navier-Stokes solver and NASTRAN has been developed and applied to a regional-jet wing design. Because the optimization system becomes very expensive computationally, only brief exploration of the design space has been performed. However, visual data mining results demonstrate that design knowledge can produce a good design even after brief design exploration.
\end{abstract}

Key Words: Aircraft, Design, Wings and Airfoil Sections, Multidisciplinary Design Optimization, Evolutionary Computation, Self-Organizing Map

\section{Introduction}

This paper discusses a new approach for multidisciplinary design optimization (MDO) by computational fluid dynamics-computational structural dynamics (CFD-CSD) coupling. MDO is a rapidly growing area of research. ${ }^{1,2)}$ Typical MDO problems involve competing objectives; for example, in aircraft design, the minimization of aerodynamic drag, minimization of structural weight, etc. While singleobjective problems may have a unique optimal solution, multi-objective problems (MOPs) have a set of compromised solutions, largely known as the trade-off surface, Pareto-optimal solutions or non-dominated solutions. These solutions are optimal in the sense that no other solutions in the search space are superior to them when all objectives are considered (Fig. 1).

Traditional optimization methods such as the gradientbased method $^{3)}$ are single-objective optimization methods that optimize only one objective. These methods usually start with a single baseline design and use local gradient information of the objective function with respect to changes in the design variables to calculate a search direction. When these methods are applied to a MOP, the problem is transformed into a single-objective optimization problem by combining multiple objectives into a single objective typically using a weighted sum method. For example, to minimize competing functions $f_{1}$ and $f_{2}$, these objective functions are combined into a scalar function $F$ as

$$
F=w_{1} \cdot f_{1}+w_{2} \cdot f_{2}
$$

(C) 2007 The Japan Society for Aeronautical and Space Sciences
This approach, however, can find only one of the Paretooptimal solutions corresponding to each set of the weights $w_{1}$ and $w_{2}$. Therefore, one must run many optimizations by trial and error, adjusting the weights to get uniform Pareto-optimal solutions over the potential Pareto-front. This is considerably costly in terms of computational time. What is more, there is no guarantee that uniform Pareto-optimal solutions can be obtained. For example, when this approach is applied to a MOP that has a concave trade-off surface, it converges to two extreme optimums without showing any trade-off information between the objectives (Fig. 2). To overcome these difficulties, the Normal-Boundary Intersection Method ${ }^{4)}$ and Aspiration Level Method ${ }^{5)}$ were developed.

An alternative approach to solve a MOP is to find as many Pareto-optimal solutions as possible to reveal trade-off information among different objectives. Once such solutions are obtained, Decision Maker (DM) is able to choose a final design with further considerations. Evolutionary algorithms (EAs, for examples, refer to Coello Coello ${ }^{6)}$ and Deb, K. ${ }^{7)}$ ) are particularly suited for this purpose.

EAs have been extended successfully to solve MO problems. ${ }^{8}{ }^{8)}$ EAs use a population to seek optimal solutions in parallel. This feature can be extended to seek Pareto solutions in parallel without specifying weights between the objective functions. Because of this characteristic, EAs can find Pareto solutions for various problems having convex, concave and discontinuous Pareto fronts. The resultant Pareto solutions represent global trade-offs. In addition, EAs have other advantages such as robustness and suitability for parallel computing. Due to these advantages, EAs have been 


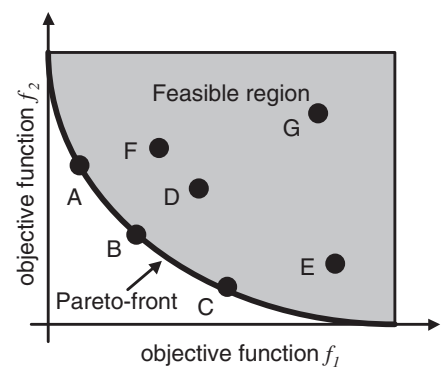

Fig. 1. The concept of Pareto-optimality.

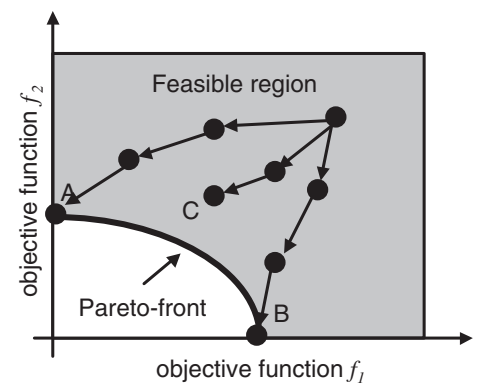

Fig. 2. Weighted-sum method applied to a MOP having a convex Pareto-front.

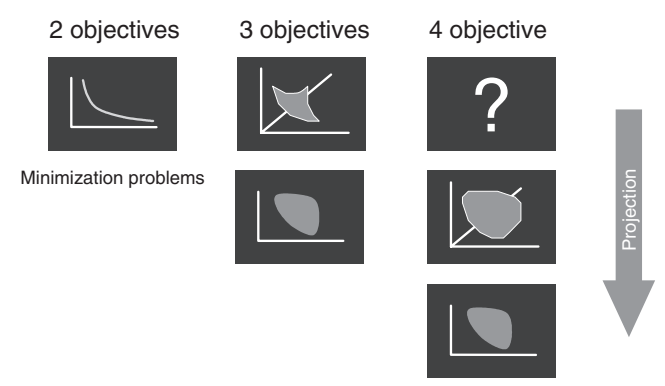

Fig. 3. Visualization of a Pareto-front.

actively applied to MOPs. ${ }^{6-9)}$ EAs have been also applied to single-objective and multi-objective aerospace design optimization problems. ${ }^{2,10-13)}$

This approach to finding many Pareto solutions works fine as it is, however, only when the number of objectives remains small (usually two, three at most, as shown in Fig. 3). To reveal trade-off information from the resultant Pareto front for real-world problems with many objectives, visualization of the Pareto front becomes an issue. Several techniques have been considered, such as parallel coordinates, ${ }^{8)}$ box plot, ${ }^{14)}$ and self-organizing maps (SOM). ${ }^{15)} \mathrm{Be}$ cause such visualization is a tool for data mining, data mining is found very important in this approach.

To support data mining activities, response surfaces are used. Once the surface is constructed, it can be used for statistical analysis; for example, analysis of variance (ANOVA). ${ }^{16)}$ ANOVA shows the effect of each design variables on objective functions quantitatively while SOM shows the information qualitatively. When the response surface method (RSM) is introduced for data mining as the post-process of optimization, it can also be applied at the pre-process stage for optimization as a surrogate model. ${ }^{17-19)}$ Pre-processing has been an important aspect in the introduction of surrogate models because it greatly reduces the computational expense, while efficiently producing rich non-dominated solutions.

As a result, the new approach for MDO, named MultiObjective Design Exploration (MODE), can be summarized as the flowchart shown in Fig. 4. MODE is not intended to give an optimal solution. MODE reveals the structure of the design space from the trade-off information and visualizes it as a panorama for DM. DM recognizes the reason for trade-offs from non-dominated designs, instead of receiving an optimal design without trade-off information. The main emphasis of the present approach is based on visual data mining.

This paper reports the results of visual data mining applied to a MDO problem for a regional-jet wing design (Fig. 5) under the small jet aircraft R\&D project sponsored by the New Energy Development Organization of Japan (NEDO). The present MDO system employs a NavierStokes (N-S) code coupled with NASTRAN. Because the resulting optimization system became very computationally expensive, RSM was not applied in this paper. EAs were applied to explore the design space briefly and then data mining was applied. The knowledge extracted from the design exploration was able to produce a better design.

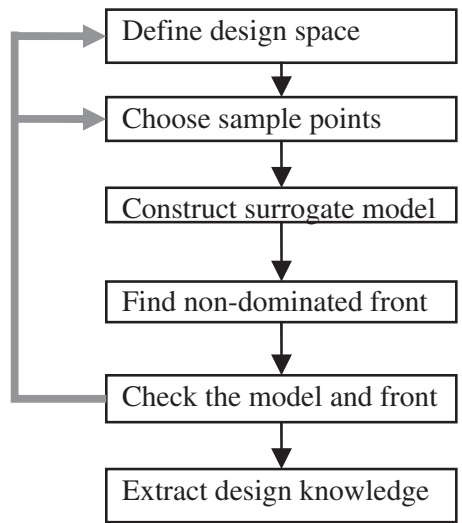

Parameterization: PARSEC, B-Spline, etc.

Design of Experiment: Latin Hypercube

Response Surface Method: Kriging Model

Optimization: Adaptive Range Multi Objective Genetic Algorithms

Uncertainty Analysis: Expected Improvement based on Kriging Model, statistics of design variables, etc.

Data Mining: Analysis of Variance, Self-Organizing Map, etc.

Fig. 4. Flowchart of Multi-Objective Design Exploration (MODE) with component algorithms. 


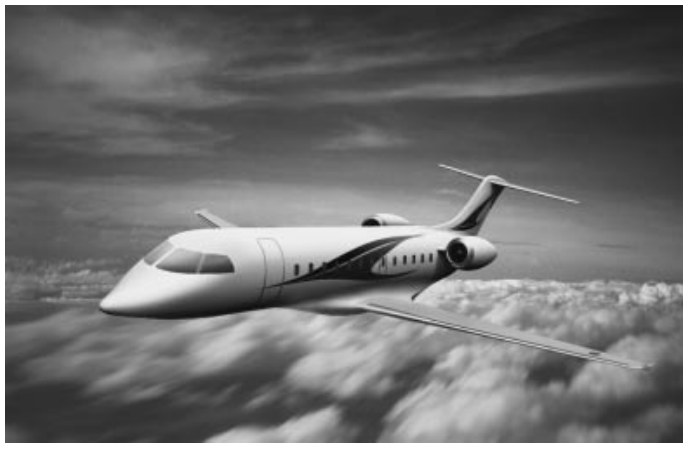

Fig. 5. Artists illustration of a regional jet (courtesy of Mitsubishi Heavy Industries, Ltd.).

\section{Multidisciplinary Wing Design}

\subsection{Objective functions}

In this optimization method, minimization of the block fuel in a required target range derived from aerodynamics and structures is considered as the primary objective function. In addition, two more objective functions are considered: minimization of the maximum take-off weight and minimization of the drag divergence between transonic and subsonic conditions.

\subsection{Geometry definition}

The design variables describe airfoil, twist, and wing dihedral. The airfoil was defined at three spanwise cross-sections using the modified PARSEC with nine design variables $\left(x_{\mathrm{up}}, z_{\mathrm{up}}, z_{\mathrm{xxup}}, x_{\mathrm{lo}}, z_{\mathrm{lo}}, z_{\mathrm{xxlo}}, \alpha_{\mathrm{TE}}, \beta_{\mathrm{TE}}\right.$, and $\left.r_{\text {LElo }} / r_{\text {LEup }}\right)$ for each cross-section as shown in Fig. 6 . The twists were defined at six spanwise locations, and then wing dihedrals were defined at kink and tip locations. The entire wing shape was thus defined using 35 design variables.

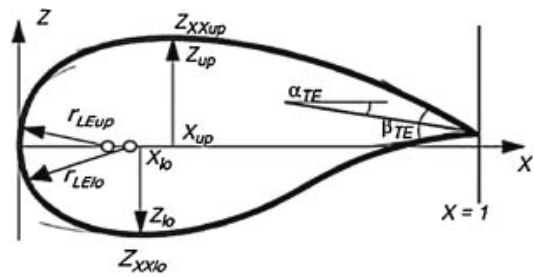

Fig. 6. Illustration of the modified PARSEC airfoil definition.

\subsection{Evaluation method}

The present optimization method evaluates the aerodynamic and structural properties of each design candidate as follows:

1. Structural optimization is performed to jig shape to realize minimum wing weight with constraints of strength and flutter requirements using NASTRAN. Then the weights of wing box and fuel carried are calculated.

2. Static aeroelastic analysis is performed for three flight conditions to determine the aeroelastic shapes (1G shape) deformation using the Euler solver and NASTRAN (Fig. 7).

3. Aerodynamic evaluations are performed for the $1 \mathrm{G}$ shapes using a N-S solver.

4. Flight envelope analysis is performed using the properties obtained as specified above to evaluate the objective functions. Using the objective functions, the optimizer generates new individuals for the next generation via genetic operations, such as selection, crossover, and mutation.

The entire flowchart is given in Fig. 8.

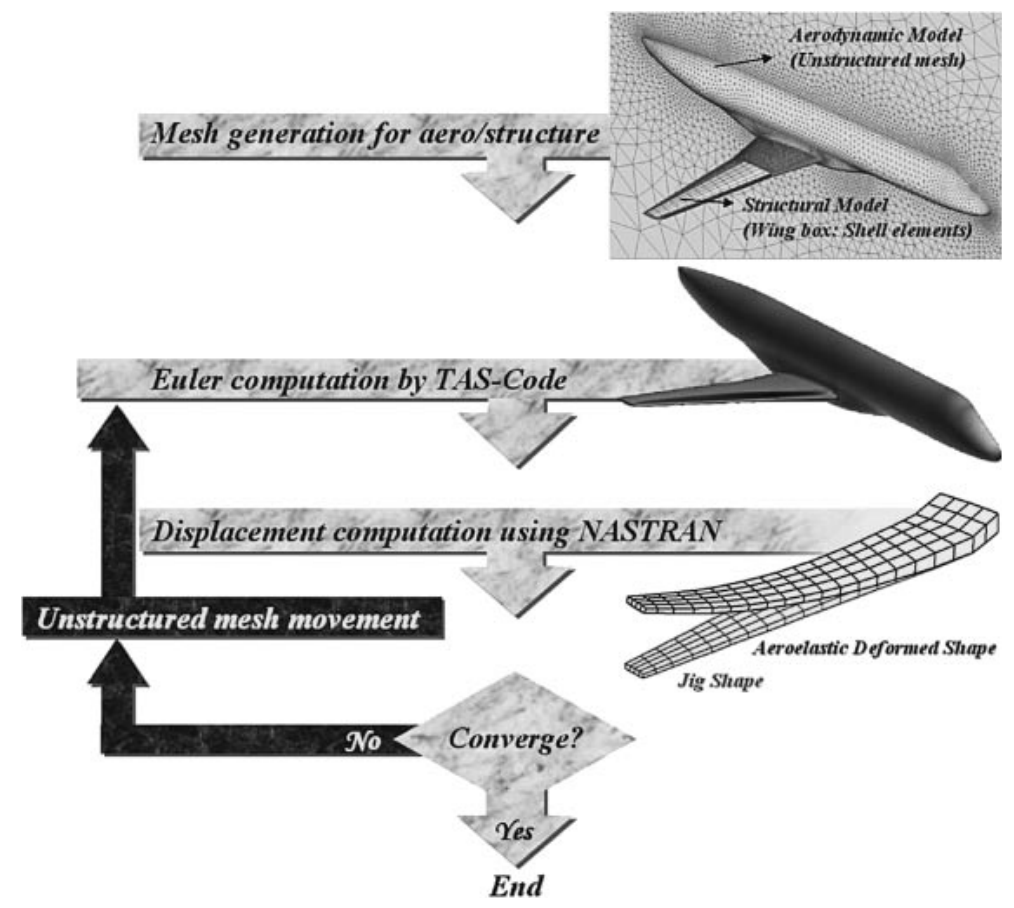

Fig. 7. Flowchart for static aeroelastic analysis. 


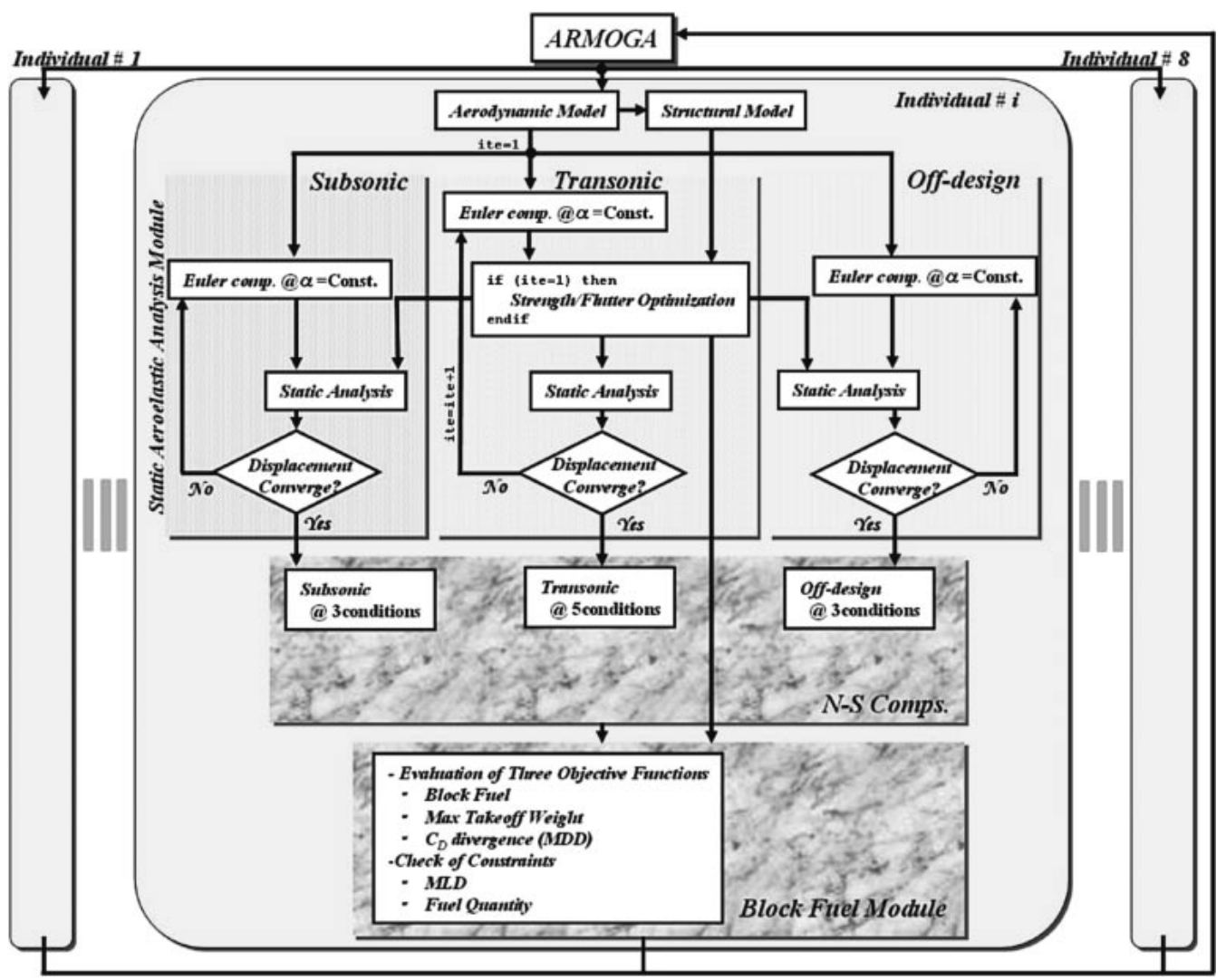

Fig. 8. Flowchart of the present MDO system for regional-jet wing design.

\section{Self-Organizing Map (SOM)}

\subsection{General SOM algorithm}

SOM is an unsupervised learning, nonlinear projection algorithm $^{20,21)}$ from high to low-dimensional space. This projection is based on the self-organization of a low-dimensional array of neurons. In the projection algorithm, the weights between the input vector and the array of neurons are adjusted to represent features of the high-dimensional data on a low-dimensional map. The closer two patterns are in the original space, the closer the response of two neighboring neurons in the low-dimensional space. Thus, SOM reduces the dimensions of input data while preserving their features.

A neuron used in SOM is associated with weight vector $\boldsymbol{m}_{i}=\left[m_{i 1}, m_{i 2}, \ldots, m_{i n}\right](i=1, \ldots, M)$, where $n$ is equal to the dimension of input vector and $M$ is the number of neurons. Each neuron is connected to adjacent neurons by a neighborhood relation and usually forms a two-dimensional rectangular or hexagonal topology as shown in Fig. 9.

The learning algorithm of SOM starts by finding the bestmatching unit $\left(\boldsymbol{m}_{\mathrm{c}}\right)$, which is closest to the input vector $\boldsymbol{x}$, as follows:

$$
\left\|\boldsymbol{x}-\boldsymbol{m}_{\mathrm{c}}\right\|=\min \left\|\boldsymbol{x}-\boldsymbol{m}_{k}\right\| \quad(k=1, \ldots, M) .
$$

Once the best-matching unit is determined, weight adjustments are performed not only for the best-matching unit, but also for its neighbors. The adjustment depends on the distance (similarity) between the input vector and the neuron.

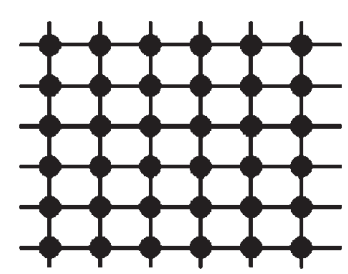

(a) Rectangular

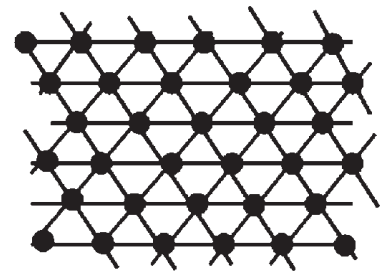

(b) Hexagonal
Fig. 9. Topology used in SOMs.

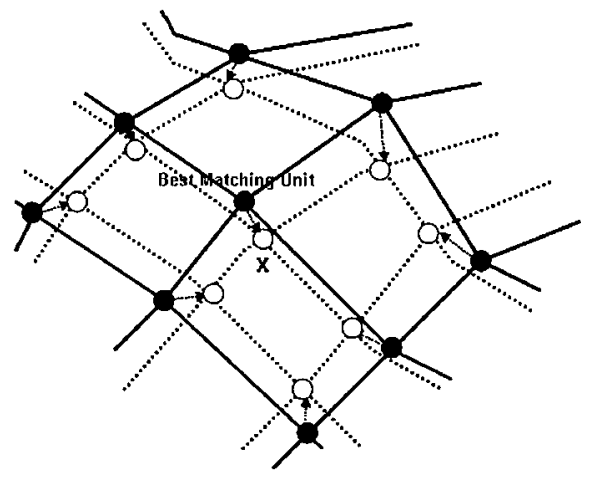

Fig. 10. Adjustment of the best-matching unit and its neighbors.

Based on the distance, the best-matching unit and neighboring units become closer to the input vector as shown in Fig. 10. The weight vectors are situated at the crossing points of the solid lines. The best-matching unit is the 
weight vector that is closest to the input vector $\boldsymbol{x}$. The bestmatching unit and its neighbors are adjusted to be closer to the input vector $\boldsymbol{x}$. The adjusted topology is represented with dashed lines. Repeating this learning algorithm, the weight vectors become smooth not only locally, but also globally. Thus, the sequence of close vectors in the original space results in a sequence of corresponding neighboring neurons in the two-dimensional map.

\subsection{Kohonen's Batch-SOM}

In this investigation, SOMs are generated using the commercial software Viscovery ${ }^{\circledR}$ SOMine plus $4.0,{ }^{22)}$ produced by Eudaptics GmbH. Although SOMine is based on the general SOM concept and algorithm, it employs an advanced variant of unsupervised neural networks (i.e., Kohonen's Batch SOM). The algorithm consists of two steps that are iterated until no more significant changes occur: search of the best-matching unit $c_{i}$ for all input data $\left\{\boldsymbol{x}_{i}\right\}$ and adjustment of weight vector $\left\{\boldsymbol{m}_{j}\right\}$ near the best-matching unit. The Batch-SOM algorithm can be formulated as follows:

$$
\begin{gathered}
c_{i}=\arg \min _{j}\left\|\boldsymbol{x}_{i}-\boldsymbol{m}_{j}\right\| \\
\boldsymbol{m}_{j}^{*}=\frac{\sum_{i} h_{j c_{i}} \boldsymbol{x}_{i}}{\sum_{i^{\prime}} h_{j c_{i^{\prime}}}}
\end{gathered}
$$

where $\boldsymbol{m}_{j}^{*}$ is the adjusted weight vector. The neighborhood relationship between two neurons $j$ and $k$ is defined by the following Gaussian-like function:

$$
h_{j k}=\exp \left(-\frac{d_{j k}^{2}}{r_{t}^{2}}\right),
$$

where $d_{j k}$ denotes the Euclidean distance between neuron $k$ and neuron $j$ on the map, and $r_{\mathrm{t}}$ denotes the neighborhood radius which is decreased with iteration steps $t$.

The standard Kohonen algorithm adjusts the weight vector after each record is read and matched. On the contrary, the Batch-SOM takes a 'batch' of data (typically all records) and performs a 'collected' adjustment of the weight vectors after all records have been matched. This is much like 'epoch' learning in supervised neural networks. The Batch-SOM is a more robust approach, since it is mediated over a large number of learning steps. For SOMine, the uniqueness of the map is ensured by the adoption of the Batch-SOM and linear initialization for input data. Much like some other SOMs, SOMine creates a map in a twodimensional hexagonal grid. Starting from numerical, multivariate data, the nodes on the grid gradually adapt to the intrinsic shape of the data distribution. Since the order on the grid reflects the neighborhood within the data, features of the data distribution can be read off from the emerging map on the grid. The trained SOM is systematically converted into visual information.

\subsection{Cluster analysis}

Once the high-dimensional data is projected on the twodimensional regular grid, the map can be used for visualization and data mining. It is efficient to group all neurons by similarity to facilitate SOM for qualitative analysis, because the number of neurons on the SOM is large as a whole. This process of grouping is called 'clustering'.

A hierarchical agglomerative algorithm is used for the clustering here. First, each node itself forms a single cluster and two clusters, which are adjacent in the map, are merged in each step. The distance between the two clusters is calculated using the SOM-ward distance. ${ }^{22)}$ The number of clusters is determined by the hierarchical sequence of clustering. A relatively small number of clusters is used for visualization, while a large number is used for the generation of weight vectors for respective design variables.

\section{Data Mining Results}

In this design, we focus on the visual data mining part of MODE. Instead of searching for the optimal solution using RSM, we have applied adaptive range multi-objective genetic algorithm (ARMOGA) to explore the design space briefly. ARMOGA generated eight individuals per generation. The optimization process was stopped when improvements were observed in all objectives. Then, SOM was applied to visualize the design space using all of the solutions computed up to that point. Based on the observation, a new wing design was proposed and the resulting wing has been confirmed to outperform the other computed solutions. This illustrates the importance of the present approach because design knowledge can produce a better design even from brief exploration of the design space.

\subsection{Optimization results}

The population size was set to eight, and then roughly 70 Euler and $90 \mathrm{~N}-\mathrm{S}$ computations were performed in one generation. It took roughly one and nine hours of CPU time per PE on SX-7 computer for single Euler and N-S computations, respectively. The population was re-initialized every five generations for the range adaptation. A total evolutionary computation of 19 generations was carried out. The evolution had not yet converged. However, the results were satisfactory because several non-dominated solutions achieved significant improvements over the initial design. Furthermore, a sufficient number of solutions are searched so that the sensitivity of the design space around the initial design can be analyzed.

Figure 11 shows all solutions projected on a two-dimensional plane between two objectives, the block fuel and the drag divergence. A non-dominated front is formed, indicating the trade-off between the block fuel and drag divergence. All solutions projected on two-dimensional planes between other combinations are shown in Figs. 12 and 13. As the non-dominated solutions did not comprise a front, these figures indicate that there are no global trade-offs between these combinations of the objective functions.

The comparison between initial and optimized geometries is investigated. Although the wing box weight tends to increase as compared to that of the initial geometry, the block fuel can be reduced. Thus, the aerodynamic performance can redeem the penalty due to the structural weight. An individual on the non-dominated front shown in 


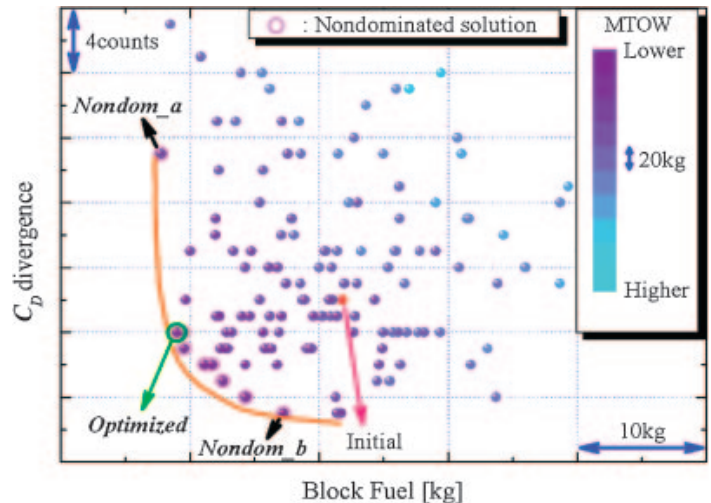

Fig. 11. All solutions between block fuel and drag divergence on a two-dimensional plane.

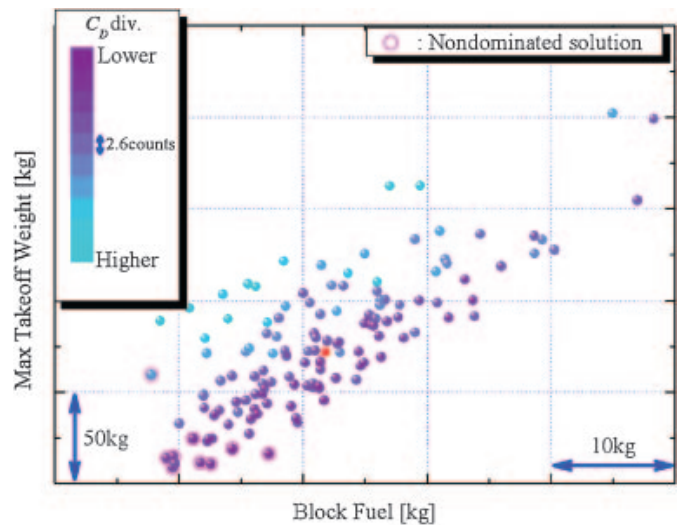

Fig. 12. All solutions between block fuel and maximum take-off weight on a two-dimensional plane.

Fig. 11 is selected, indicated as 'optimized,' and then the optimized geometry is compared with the initial geometry.

Although drag minimization is not considered here, $C_{\mathrm{D}}$ is reduced. By comparison of the polar curves at constant $C_{\mathrm{L}}$ for the cruising condition, the $C_{\mathrm{D}}$ of the optimized geometry is found to be reduced by 5.5 counts. Due to the improvement in drag, the block fuel of the optimized geometry is decreased by more than $1 \%$ even with its structural weight penalty.

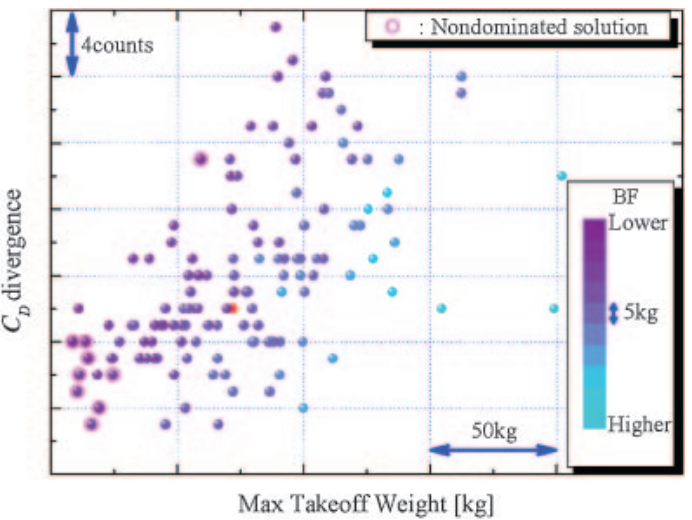

Fig. 13. All solutions between maximum take-off weight and drag divergence on a two-dimensional plane.

\subsection{Data mining using SOM}

A detailed flow visualization for the optimized geometry indicates that the main drag reduction is achieved at the kink location. However, the optimized geometry has an inverted gull at the kink. Figure 14(a) shows the SOM colored by the angle between inboard and outboard on the upper wing surface of the gull-wing at the kink location. Angles greater and less than $180 \mathrm{deg}$ correspond to gull and inverted gull wings, respectively. Higher values of this angle, as shown in Fig. 14(a), correspond to higher $C_{\mathrm{D}}$ at the transonic cruising flight condition as shown in Fig. 14(b). However, at angles less than $180 \mathrm{deg}$, there is little correlation between Figs. 14(a) and 14(b). The inverted gull did not affect aerodynamic performance very much.

Furthermore, SOM also shows that the higher angles shown in Fig. 14(a) correspond to higher maximum takeoff weights as shown in Fig. 14(c). The inverted gull wing is known to have a structural weight increase, which is also observed in the present results. From visualization of the design space by SOM, it is suggested that non-gull wings should be designed even though the optimized geometry is an inverted gull design.

Various SOM colorings are currently compared by human inspection. This is, in fact, an advantage of SOM, because humans are good at pattern recognition. However, this could

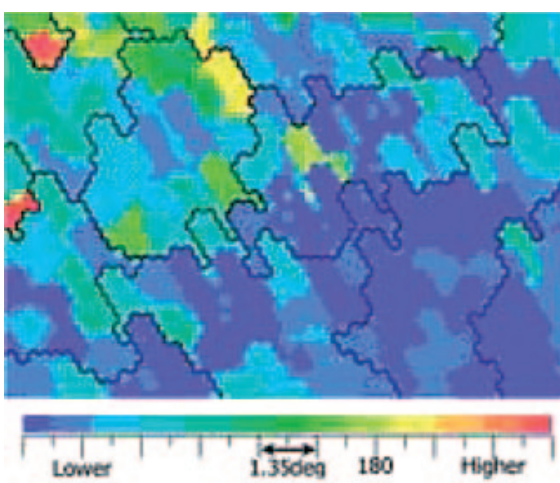

(a)

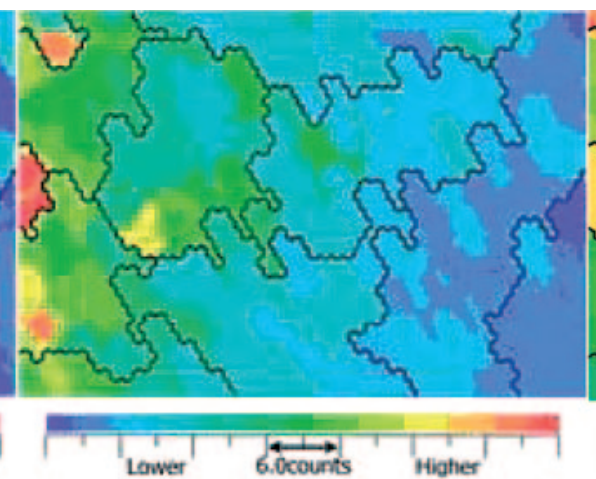

(b)

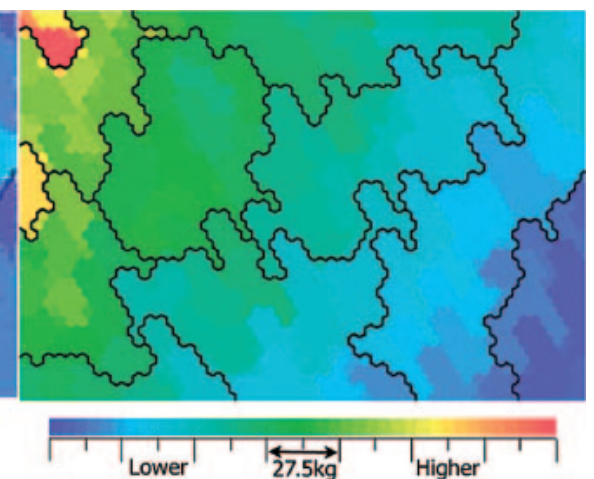

(c)

Fig. 14. SOM; (a) colored at the angle on the upper surface expressing the gull wing at the kink location, (b) colored at the CD under transonic flight cruising conditions, (c) colored at the maximum take-off weight. 


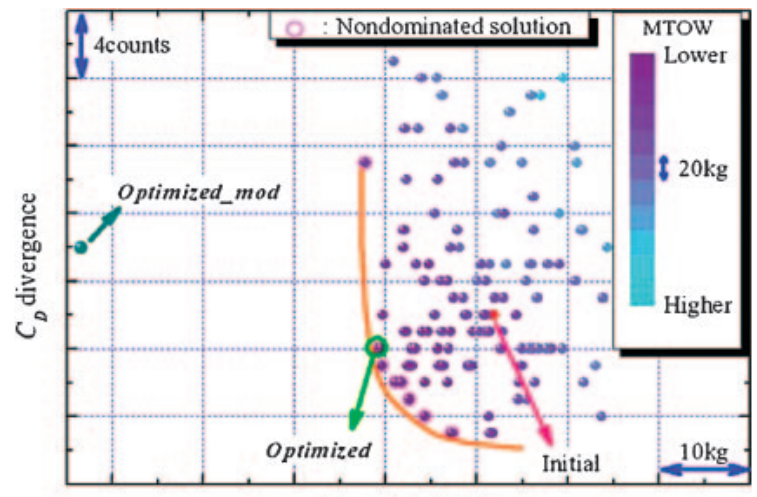

Block Fuel [kg]

Fig. 15. Comparison of optimized_mod and all solutions between block fuel and CD divergence on a two-dimensional plane.

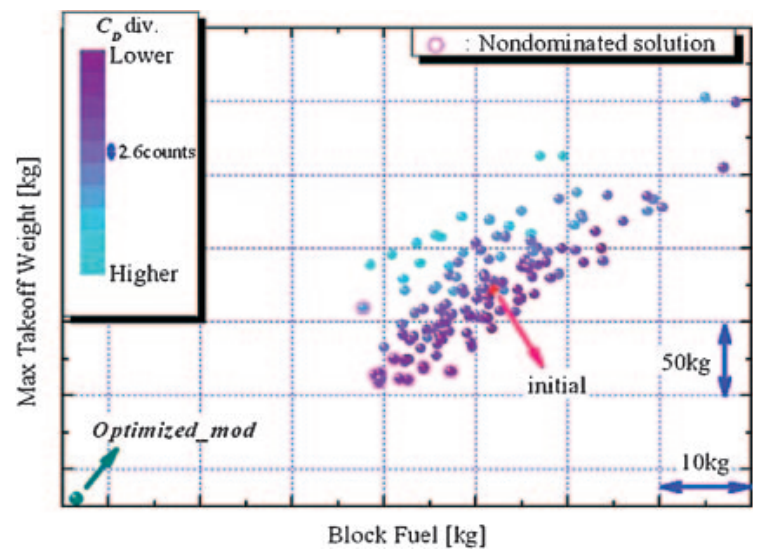

Fig. 16. Comparison of optimized_mod and all solutions between block fuel and maximum take-off weight on a two-dimensional plane.

be a potential drawback of the SOM approach if a very large number of color maps have to be inspected. To focus on a small number of important maps, other tools may be required. ANOVA has been found to compensate this potential drawback by providing the relative importance of design variables. ${ }^{17)}$ Further research will be required for efficient data mining.

\subsection{Evaluation of the non-gull geometry}

The optimized wing shape was been modified to examine whether or not the non-gull wing shape (called 'optimized_mod') can achieve better performance, and to verify the design knowledge obtained by the previous data mining.

The results are shown in Figs. 15 to 17. These figures show that the optimized_mod improves both block fuel and maximum take-off weight. Moreover, by comparison of the polar curves at constant $C_{\mathrm{L}}$ for cruising condition shown in Fig. 18, the $C_{\mathrm{D}}$ of optimized_mod is found to be reduced by 10.6 counts over the initial geometry. Due to the improvement of drag, the block fuel of optimized_mod is reduced by $3.6 \%$.

The present optimization method is probably incomplete because only a small number of generations was performed. In addition, the automatic mesh generator may severely clip the design space. In the present MDO system, surface spline

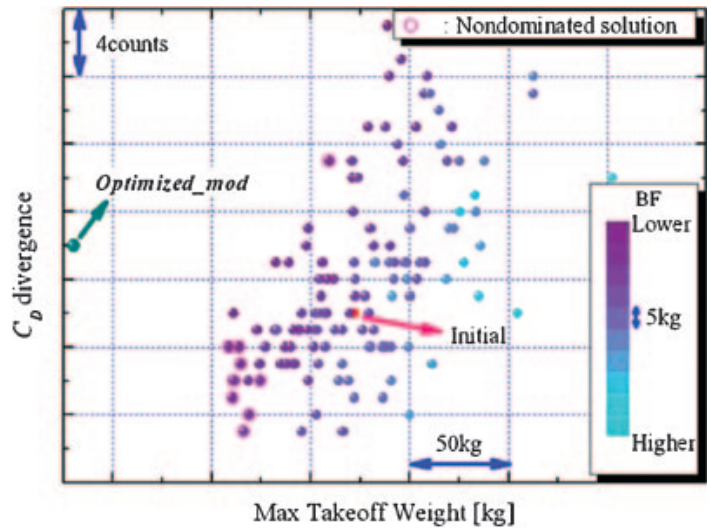

Fig. 17. Comparison of optimized_mod and all solutions between maximum takeoff weight and $C_{\mathrm{D}}$ divergence on a two-dimensional plane.

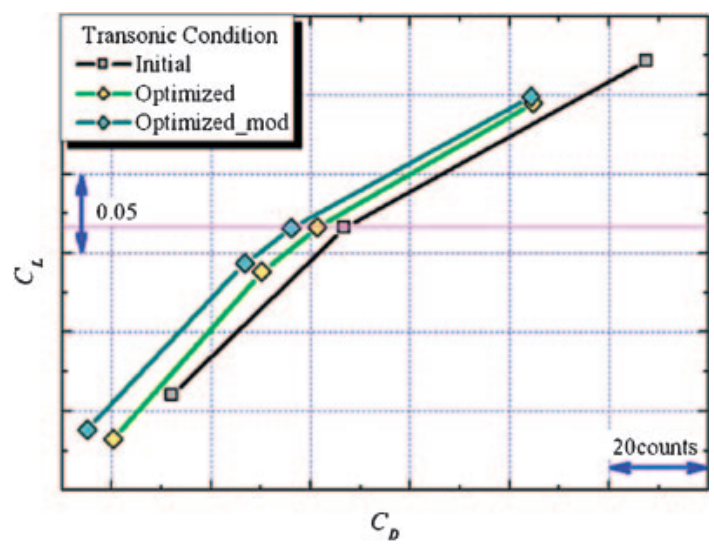

Fig. 18. Comparison of the $C_{\mathrm{L}}-C_{\mathrm{D}}$ curves among the three geometries as initial, optimized, and optimized_mod under transonic flight conditions.

function of geometry deviation is used for modifying the wing surface mesh, and then the volume mesh is modified accordingly using the unstructured dynamic mesh method. However, this process distorted the surface mesh around the leading edge. This mesh generation might be the primary reason for the difficulty in finding the non-gull geometry. However, the present results demonstrate that data mining can produce a good design even from the results of incomplete optimization.

\section{Concluding Remarks}

A new approach, MODE, has been presented to address MDO problems. MODE is not intended to give an optimal solution. MODE reveals the structure of the design space from trade-off information and visualizes it as a panorama for DM. DM recognizes the reason for trade-offs from non-dominated designs, instead of receiving an optimal design without trade-off information.

The main emphasis of this approach is visual data mining. The data mining results are presented for a high-fidelity MDO problem of a regional-jet wing design. It optimizes aerodynamic performance and structural weight under aeroelastic constraints. Because the design space was large 
and high-fidelity simulation codes were time-consuming, ARMOGA was used to briefly explore the design space. Optimization was stopped after improvements were obtained. Then SOM was applied to visualize the design space. Based on the observation, a new, better wing design was proposed. This illustrates the importance of the present approach because design knowledge can produce a better design even after only brief exploration of the design space.

Although it is not discussed in this paper, the flowchart of MODE shown in Fig. 4 has feedback loops. The design space can be redefined by analyzing the surrogate model. ${ }^{16)}$ Moreover, from data mining, competing objectives and active constraints can be identified. This will lead to re-defining the MDO problem itself. MDO often uses conceptual performance equations as design objectives. However, the sensitivity of those equations to high-fidelity simulation codes is not well understood. As more and more high-fidelity simulation codes become available to MDO, the selection of objective functions will become more crucial.

\section{Acknowledgments}

The authors would like to acknowledge the Advanced Fluid Information Research Center, Institute of Fluid Science, Tohoku University, for providing the computer resources. Part of this research was sponsored by the New Energy Development Organization of Japan (NEDO) under a small jet aircraft R\&D project. This work was also supported by No. 15206091, Grants-In-Aid for Scientific Research, Japan.

\section{References}

1) Queipo, N. V., Haftka, R. T., Shyy, W., Goel, T., Vaidyanathan, R. and Tucker, P. K.: Surrogate-Based Analysis and Optimization, Prog. Aerospace Sci., 41 (2005), pp. 1-28.

2) Sasaki, D. and Obayashi, S.: Efficient Search for Trade-Offs by Adaptive Range Multi-objective Genetic Algorithms, J. Aerospace Comput. Inform., Commun., 2 (2005), pp. 44-64.

3) Vanderplaats, G. N.: Numerical Optimization Techniques for Engineering Design, McGraw-Hill Book Company, New York, 1984.

4) Das, I. and Dennis, J. E.: Normal-Boundary Intersection: A New Method for Generating the Pareto Surface in Nonlinear Multicriteria Optimization Problems, SIAM J. Optim., 8 (1998), pp. 631-657.

5) Nakayama, H.: Aspiration Level Approach to Interactive MultiObjective Programming and Its Applications, Paldalos, P. M., Siskos, Y. and Zopounidis, C. (eds.), Advances in Multicriteria Analysis, Kluwer Academic Publishers, Dordrecht, 1995, pp. 147-174.

6) Coello Coello, A. C. and Lamont, G. B.: Applications of Multi-
Objective Evolutionary Algorithms, World Scientific Publishing Co. Pte. Ltd., Singapore, 2004.

7) Deb, K.: Multi-Objective Optimization Using Evolutionary Algorithms, John Wiley \& Sons, Ltd., New York, 2001.

8) Fleming, P. J., Purshouse, R. C. and Lygoe, R. J.: Many-Objective Optimization: An Engineering Design Perspective, Evolutionary Multi-Criterion Optimization (EMO2005), Springer, Heidelberg, 2005, pp. 14-32.

9) Fonseca, C. M. and Fleming, P. J.: Genetic Algorithms for MultiObjective Optimization: Formulation, Discussion and Generalization, Proceedings of the Fifth International Conference on Genetic Algorithms, Morgan Kaufmann Publishers, Inc., San Mateo, CA, 1993, pp. 416-423.

10) Chiba, K., Obayashi, S., Nakahashi, K. and Morino, H.: High-Fidelity Multidisciplinary Design Optimization of Aerostructural Wing Shape for Regional Jet, AIAA Paper 2005-5080, June 2005.

11) Giannakoglou, K. C.: Design of Optimal Aerodynamic Shapes Using Stochastic Optimization Methods and Computational Intelligence, Prog. Aerospace Sci., 38 (2002), pp. 43-76.

12) Oyama, A., Obayashi, S. and Nakamura, T.: Real-Coded Aptive Range Genetic Algorithm Applied to Transonic Wing Optimization, Appl. Soft Comput., 1 (2001), pp. 179-187.

13) Parussini, L., Pediroda, V. and Obayashi, S.: Design under Uncertainties of Wings in Transonic Field, JSME Int. J., Ser. B, 48 (2005), pp. 218-228.

14) Parmee, I. C. and Abraham, J. A. R.: Supporting Implicit Learning via the Visualisation of COGA Multi-objective Data, Proceedings of IEEE Congress on Evolutionary Computation, 2004, pp. 395-402.

15) Obayashi, S. and Sasaki, D.: Visualization and Data Mining of Pareto Solutions Using Self-Organizing Map, Evolutionary Multi-Criterion Optimization (EMO2003), LNCS 2632, Springer, Heidelberg, 2003, pp. 796-809.

16) Jeong, S., Chiba, K. and Obayashi, S.: Data Mining for Aerodynamic Design Space, AIAA Paper 2005-5079, June 2005.

17) Giannakoglou, K. C., Giotis, A. P. and Karakasis, M. K.: Low-Cost Genetic Optimization Based on Inexact Pre-Evaluations and the Sensitivity Analysis of Design Parameters, J. Inverse Prob. Eng., 9 (2001), pp. 389-412.

18) Keane, A. J.: Wing Optimization Using Design of Experiment, Response Surface, and Data Fusion Methods, J. Aircraft, 40 (2003), pp. 741-750.

19) Willson, B., Cappelleri, D., Simpson, T. W. and Frecker, M.: Efficient Pareto Frontier Exploration Using Surrogate Approximations, Optim. Eng., 2 (2001), pp. 31-50.

20) Kohonen, T.: Self-Organizing Maps, Springer, Berlin, Heidelberg, 1995.

21) Krzysztof, J. C., Witold, P. and Roman, W. S.: Data Mining Methods for Knowledge Discovery, Kluwer Academic Publisher, Boston, 1998.

22) Eudaptics software gmbh, http://www.eudaptics.com/somine/, last access on April 14, 2005.

23) Donald, R. J., Matthias, S. and William, J. W.: Efficient Global Optimization of Expensive Black-Box Function, J. Global Optim., 13 (1998), pp. 455-492.

24) Jeong, S., Murayama, M. and Yamamoto, K.: Efficient Optimization Design Method Using Kriging Model, J. Aircraft, 42 (2005), pp. 413-420. 